\title{
THE ROLE OF DISTRIBUTION AND VOLATILITY SPECIFICATION IN VALUE AT RISK ESTIMATION: EVIDENCE FROM THE JOHANNESBURG STOCK EXCHANGE
}

\author{
John Muteba Mwamba \\ University of Johannesburg \\ johnmu@uj.ac.za
}

\author{
Kruger Pretorius+ \\ University of Johannesburg \\ 201041819@student.uj.ac.za
}

July 2011

\begin{abstract}
Given the volatile nature of global financial markets, managing as well as predicting financial risk plays an increasingly important role in banking and finance. The Value at Risk (VaR) measure has emerged as the most prominent measure of downside market risk. It is measured as the alpha quantile of the profit and loss distribution. Recently a number of distributions have been proposed to model VaR: these include the extreme value theory distributions (EVT), Generalized Error Distribution (GED), Student's t, and normal distribution. Furthermore, asymmetric as well as symmetric volatility models are combined with these distributions for out-sample VaR forecasts. This paper assesses the role of the distribution assumption and volatility specification in the accuracy of VaR estimates using daily closing prices of the Johannesburg Stock Exchange All Share Index (JSE ALSI). It is found that Student's $t$ distribution combined with asymmetric volatility models produces VaR estimates in out-sample periods that outperform those from models stemming from normal, EVT/symmetric volatility specification.
\end{abstract}

Keywords

Value at Risk, asymmetric GARCH, Extreme Value Theory, violations

\footnotetext{
\#Dr John Muteba Mwamba is a senior lecturer at the Department of Economics \&Econometrics, University of Johannesburg, South Africa.

+Mr Kruger Pretorius is a master's student in Financial Economics at the Department of Economics \&Econometrics, University of Johannesburg, South Africa.
} 


\section{INTRODUCTION}

The measurement of market risk has become a primary concern for market regulators and financial institutions. This is largely due to the increased volatility and interdependence in global financial markets in recent years. Market risk refers to the risk of incurring trading book losses caused by adverse movements in market prices and returns. Banks are particularly affected by such risks, due to the establishment of the Basel Capital Accord (Basel I) in 1996 and more recently Basel III in 2010. The Capital Adequacy Directive by the Bank of International Settlement in Basel requires internationally active banks to hold sufficient risk capital to cover possible losses on their trading portfolios on $99 \%$ of occasions, over a holding period of ten days (Frey \& McNeil, 2000). The value of such a capital requirement is termed VaR. Specifically VaR refers to the magnitude of likely losses over a specified period, resulting from 'normal' market movements.

Intuitively VaR is measured as the alpha quantile of the return distribution of a portfolio. The assumption made about the observed return distribution is key to VaR computation. The list of distributions used in empirical applications includes the EVT, GED, Student's t, and normal distribution. This paper assesses the role of the distribution assumption and volatility specification in the accuracy of VaR estimates using daily closing prices of the JSE ALSI. Contrary to many studies (Fernandez, 2003; Frey and McNeil, 2000) that recommend the use of EVT to model the fat-tailed behaviour of return distributions in VaR computations, our results show that the Student's $t$ distribution combined with asymmetric volatility models produces VaR estimates in out-sample periods that outperform VaR estimates obtained with models stemming from normal, EVT/symmetric volatility specification. These results suggest that the returns on the JSE are more leptokurtic with few occurrences of extreme losses.

Distribution assumption, volatility specification and their implications for VaR estimates are an issue that has been also investigated recently by Huang, Hung and Chang (2010). They examine the relative importance of both the distribution assumption as well as variance specification in parametric VaR estimation, and find that both play significant roles. For the volatility specification in the parametric variance-covariance approach, for instance, they find that specifying either a symmetric, or alternatively an asymmetric volatility process can have a significant impact on the VaR results. Similarly, Burns (2002) finds that the quality of the VaR estimate depends solely on the type of GARCH model employed in the returns volatility specification. It is worth noting that a symmetric GARCH process assumes that positive and negative market shocks have impacts of similar magnitude on return volatility. In practice, however, negative shocks are found to have larger impact than positive shocks on the volatility of financial returns.

Moreover, in an empirical comparison of different approaches for calculating and predicting VaR, Kuester, Mittnik and Paolella (2006) find that parametric VaR methods perform less well than is desirable from a statistical perspective due to distributional assumptions imposed on observed return distribution. Financial returns exhibit volatility clustering, excess kurtosis and skewness. As a result, standard methods of VaR calculation, in which it is assumed beforehand that a given distribution (normal, Student's t, EVT, GED etc.) obtains, produce different results. This paper aims at finding the best combination of distribution and volatility specification that produces the best and most reliable VaR estimates. The paper proceeds as follows: section 2 describes VaR and volatility specifications, followed by distribution assumptions; and section 3 presents the empirical results and conclusion. 


\section{METHODOLOGY}

Traditionally, the volatility dynamics of financial returns are accurately modelled by employing the GARCH and/or Exponential GARCH (EGARCH) models proposed by Bollerslev (1986) and Nelson (1991) respectively. Two main motivations exist for using GARCH type models for the financial return volatility process. Firstly, the approximation-theoretical properties of the models are attractive, as they are able to describe the volatility clustering and leptokurtosis (high peaks and fat tails) that are observed in high frequency financial data. Secondly, GARCH models focus on the conditional variance rather than the unconditional variance of time series. From an economic perspective this is appropriate, since any uncertainty that can be eliminated by relying on past values is irrelevant (Kuester et al., 2006)

The $\operatorname{GARCH}(1,1)$ model is most commonly employed in the literature. The process is specified by a mean equation and variance equation as follows:

$$
\begin{array}{ll}
\text { Mean equation: } & r_{t}=\mu\left(r_{t} / r_{t-1}\right)+\sigma_{t} z_{t} \\
z \sim N(0,1), & \\
\text { Variance equation: } & \sigma_{t}^{2}=\alpha_{0}+\alpha e_{t}^{2}+\beta \sigma_{t-1}^{2}
\end{array}
$$

where $\alpha_{0}, \alpha$, and $\beta$ are volatility parameters to be estimated. These three parameters capture the volatility dynamics of the conditional mean $\mu\left(r_{t} / r_{t-1}\right)$. The GARCH $(1,1)$ process is stationary if $(\alpha+\beta)<1$. The return-generating process is specified in the mean equation. Even in cases where this equation does not provide an optimal specification of the return-generating process, Nelson (1992) finds that GARCH models are robust to misspecification. He concludes that the GARCH model is able to estimate the volatility process in a consistent manner, despite misspecification of the mean equation. Of greater importance is the specification of the variance equation, i.e. the volatility process.

An alternative form of the GARCH model is the Exponential GARCH (EGARCH) model (Nelson, 1991). The EGARCH model addresses a significant shortcoming of the GARCH model, namely its inability to capture the leverage effect. The leverage effect refers to the possibility that downward movements in the market are followed by higher volatilities than upward movements of the same magnitude. In this regard, the GARCH model is known as an example of symmetric volatility specification, whereas the EGARCH model is an example of an asymmetric volatility specification. Another advantage of the EGARCH model is that no restrictions need to be imposed on the volatility process for the purposes of estimation. This is in contrast to the stationary parameter restrictions of the GARCH model. The EGARCH model is described as nonlinear - i.e. the conditional variance is no longer modelled as a linear function of the lagged variance and lagged squared residual.

The variance specification in the EGARCH model takes the form:

$$
\log \left(\sigma_{t}^{2}\right)=\alpha_{0}+\beta \cdot \log \left(\sigma_{t-1}^{2}\right)+\alpha\left|\frac{e_{t-1}}{\sigma_{t-1}}\right|+\gamma \frac{e_{t-1}}{\sigma_{t-1}}
$$

In this EGARCH model, the conditional variance is captured in its logarithmic form. This implies that the leverage effect is exponential, which ensures that forecasts of the conditional variance will be non-negative. The leverage effect is captured by the coefficient $\gamma$. 


\section{DISTRIBUTION ASSUMPTIONS}

GARCH models assume that the underlying return-generating process has a normal distribution. In practice, financial returns (and innovations) are not normally distributed (Muteba Mwamba, 2011). The normal distribution is a symmetrical distribution that can be fully described by its mean and standard deviation. In relation to financial returns, the normal distribution assumption has one major flaw: it drastically understates the size as well as frequency of extreme events (Urbani, 2010). This is due to the fact that the ends of the normal distribution tail off too quickly to be able to accurately describe the size and frequency of extreme events. Most financial market indices exhibit leptokurtosis (excess kurtosis) as well as negative skewness in their distributions. Leptokurtosis refers to the property that the distribution is more peaked than the normal distribution and has fatter tails than the normal distribution. The distribution's negative skewness refers to the fact that there are more downside return extremes than upside return extremes. As Urbani (2010) notes, it is this negative skewness of the return distribution that explains the asymmetry of returns. Estimating VaR measures under the incorrect normality assumption significantly underestimates the frequency and size of extreme events.

\section{MARKET RISK MODEL}

To implement a robust market risk model that reliably predicts potential losses in the Johannesburg Stock Exchange, we first fit the return series by the following process:

$$
r_{t}=\mu\left(r_{t} / r_{t-1}\right)+\sigma_{t} z_{t}
$$

where $\boldsymbol{r}_{\boldsymbol{t}}, \boldsymbol{\mu}_{\boldsymbol{t}}\left(\boldsymbol{r}_{\boldsymbol{t}} / \boldsymbol{r}_{\boldsymbol{t}-1}\right), \boldsymbol{z}_{\boldsymbol{t}}, \boldsymbol{\sigma}_{\boldsymbol{t}}$ represent respectively the return series, the conditional mean return, assumed to be an $\operatorname{AR}(1)$, the conditional volatility, and the innovations, assumed to be independent and identically distributed with mean zero and unit variance. The following equation is used to forecast the one-step-ahead forecast of VaR estimates:

$$
\operatorname{VaR}_{t+1}(\propto)=\mu_{t+1}\left(r_{t} / r_{t-1}\right)+\sigma_{t+1}\left(r_{t} / r_{t-1}\right) F^{-1}(\alpha)
$$

where $\boldsymbol{F}^{-1}(\boldsymbol{\alpha})$ is the alpha quantile of the innovations conditional distribution. We use both GARCH and EGARCH processes to model current volatility $\left(\boldsymbol{\sigma}_{t}\right)$ in Equation (5) and assume that the innovations conditional distribution $\boldsymbol{F}^{\mathbf{- 1}}(\boldsymbol{\alpha})$ is normal, Student's t and Generalized Pareto Distribution (GPD) respectively. The assumption we make about the innovation conditional distribution $\boldsymbol{F}^{-1}(\boldsymbol{\alpha})$ allows us to compare separately EVT-based VaR estimates with those obtained with normal or Student's t distribution.

To understand the implications of the distribution assumption about innovations conditional distribution $\boldsymbol{F}^{-\mathbf{1}}(\boldsymbol{\alpha})$, we use different combinations of normal, Student's t and GPD distribution with both symmetric $(\mathrm{GARCH})$ and asymmetric $(\varepsilon G A R C H)$ processes. These combinations yield the following six different market risk models using Equation (5) above:

$$
\begin{array}{lll}
\mathrm{GARCH} & -\mathrm{N} & \text { (symmetric GARCH, normally distributed innovations) } \\
\mathrm{GARCH} & -\mathrm{T} & \text { (symmetric GARCH, Student's tinnovations) } \\
\mathrm{EGARCH}-\mathrm{N} & \text { (asymmetric GARCH, normally distributed innovations) }
\end{array}
$$




$$
\begin{array}{lll}
\text { EGARCH } & -\mathrm{T} & \text { (asymmetric GARCH, Student's t innovations) } \\
\mathrm{GARCH} & -\mathrm{GPD} & \text { (symmetric GARCH, Generalized Pareto innovations) } \\
\mathrm{EGARCH} & -\mathrm{GPD} & \text { (asymmetric GARCH, Generalized Pareto innovations) }
\end{array}
$$

\section{STRESS-TESTING THE MODELS}

Equation (5) is used to obtain a one-period-ahead forecast of $V a R$ in the out-sample period. Both upside and downside VaR forecasts are estimated. Stress testing is practically based on counting the number of violations for each one of the six market risk models abovementioned. Our aim is to see how well assumed innovations conditional distribution (normal, Student's t or EVT) fits individually both the upper and lower tails of observed return distribution. In this case, a violation refers to an instance where an actual observed JSE ALSI loss (gain) exceeds the forecasted downside (upside) VaR estimate as measured by one of the six abovementioned market risk models. The intuition behind counting violations is straightforward: over the period under consideration, the market risk model that results in few violations will be deemed the best market risk model.

\section{EMPIRICAL RESULTS}

Daily JSE All Share Index (ALSI) price data for the period between 31 December 2000 and 29 July 2010 is employed, making up a total of 2759 observations. We divide this sample into two arbitrary sub-samples: the in-sample period (31 December 2000 to 02 September 2007) and the out-sample period ( 03 September 2007 to 29 July 2010). Our objective is to compare the ability of each one of the abovementioned market risk models in forecasting the JSE ALSI potential losses in the out-sample period. The ALSI returns are generated from the price data as follows:

$$
\operatorname{RETALSI} I_{t}=\ln \left(P_{t}\right)-\ln \left(P_{t-1}\right)
$$

where $\boldsymbol{R E T A L S \boldsymbol { I } _ { \boldsymbol { t } }}$ represents period $\boldsymbol{t}$ return, $\boldsymbol{P}_{\boldsymbol{t}}$ represents period $\boldsymbol{t}$ price, and $\boldsymbol{P}_{\boldsymbol{t}-\mathbf{1}}$ is price in period $\boldsymbol{t}-\mathbf{1}$. TABLE $\mathbf{l}$ shows that the daily returns series exhibits negative skewness as well as excess kurtosis, and that the Jarque Bera normality test is rejected. Clearly, the ALSI daily return series is not normally distributed.

TABLE 1: Descriptive statistics

\begin{tabular}{cccccc}
\hline Mean & Stddev & Skewness & Kurtosis & Jarque Bera & Pbty of JB \\
\hline 0.00045 & 0.01324 & -0.20612 & 6.41433 & 1359.68300 & 0.00000 \\
\hline
\end{tabular}

Source: Authors'calculations

The conditional mean is found to be an AR (3) given that it has the lowest Akaike Information Criterion (AIC) when compared with other lag specifications. The AIC makes adjustments to the likelihood function in order to account for the number of parameters to be estimated.

Estimation results for the six market risk models are displayed in TABLE 2. The symmetric GARCH $(1,1)$ models all satisfy the GARCH parameter restrictions; all the parameters in the variance equation are statistically significant. Also, $(\alpha+\beta)<1$, indicating that the symmetric GARCH 
processes are stationary. $(\alpha+\beta)$ determines how quickly the variance forecast will converge to the unconditional variance.

In the EGARCH models, all estimated parameters in the variance equations were found to be statistically significant. The EGARCH models can incorporate the leverage effect. This is because the conditional variance equations in the $\operatorname{GARCH}(1,1)$ models depend only on the magnitudes of the lagged residuals, and ignore their signs. The leverage effect term in the EGARCH model is represented by RESID $(-1) / S P R T$ (GARCH $(-1)$ ). For the leverage effect to be present, this estimated parameter is expected to be negative and statistically significant, as is found to be the case.

The AIC and Schwartz Bayesian Criterion (SBC) are used to select the volatility model that provides the best fit for the data. The AIC and $S B C$ for the six estimated models are also displayed in TABLE 2. The EGARCH-T model has the lowest AIC and SBC statistics, indicating that it provides the best fit for the data.

For the GARCH-GPD and EGARCH-GPD models, the estimated parameters of the Generalized Pareto Distribution are also displayed. These are the threshold $U$, the shape parameter $\xi$ and the scale parameter $\beta$ used for the quantile calculation.

TABLE 2: Distribution and GARCH type results

\begin{tabular}{|c|c|c|c|c|c|c|}
\hline & GARCH-N & GARCH-T & $\begin{array}{c}\text { EGARCH- } \\
\quad N \\
\end{array}$ & $\begin{array}{c}\text { EGARCH- } \\
\quad T \\
\end{array}$ & $\begin{array}{l}\text { GARCH- } \\
\text { GPD }\end{array}$ & $\begin{array}{l}E G A R C H \\
-G P D\end{array}$ \\
\hline \multicolumn{7}{|l|}{ Mean $\varepsilon q$. } \\
\hline Constant & $0.0008 *$ & $0.0009 *$ & $0.0005 *$ & $0.0006 *$ & $0.0009 \star$ & $0.0006 *$ \\
\hline $\operatorname{AR}(1)$ & $0.0657^{\star}$ & $0.0610 *$ & $0.0575 *$ & $0.0578 *$ & $0.0619 *$ & $0.0573 *$ \\
\hline $\operatorname{AR}(2)$ & 0.0245 & 0.0226 & 0.0134 & 0.0146 & 0.0245 & 0.0147 \\
\hline $\operatorname{AR}(3)$ & $-0.0566 \star$ & $-0.0540 \star$ & $-0.057 \star$ & $-0.053 \star$ & $-0.0553 \star$ & $-0.056 *$ \\
\hline \multicolumn{7}{|l|}{ Variance $\varepsilon q$. } \\
\hline Constant & $0.000003 *$ & $0.000003 *$ & $-0.335 \star$ & $-0.304 \star$ & $0.000003 *$ & $-0.326 *$ \\
\hline $\operatorname{Resid}(-1)^{\wedge} 2[\alpha]$ & $0.1123 *$ & $0.1064^{\star}$ & & & $0.1096 *$ & \\
\hline $\operatorname{GARCH}(-1)[\beta]$ & $0.8713 *$ & $0.8763 \star$ & & & $0.8733^{\star}$ & \\
\hline Student's t dof & & $14.16^{*}$ & & $15.31 *$ & & \\
\hline GED parameter & & & & & $1.71^{\star}$ & $1.74 \star$ \\
\hline $\begin{array}{l}\text { ABS }[\operatorname{Resid}(-1) / \text { sqrt } \\
\text { (GARCH(-1)] }\end{array}$ & & & $0.1739 *$ & $0.1516 *$ & & $0.1671 *$ \\
\hline $\begin{array}{l}\operatorname{Resid}(-1) / \text { sqrt } \\
(\operatorname{GARCH}(-1))\end{array}$ & & & $-0.079 \star$ & $-0.084 *$ & & $-0.080 \star$ \\
\hline $\operatorname{LOG}(\operatorname{GARCH}(-1))$ & & & $0.9779 \star$ & $0.9795^{\star}$ & & $0.9784 \star$ \\
\hline$\alpha+\beta$ & 0.983 & 0.982 & & & 0.983 & \\
\hline AIC & -6.0795 & -6.0875 & -6.0945 & -6.102 & -6.0848 & -6.0982 \\
\hline SBC & -6.0644 & -6.0703 & -6.0773 & -6.0827 & -6.0676 & -6.0789 \\
\hline Quantile & 2.33 & 2.97 & 2.33 & 2.94 & 2.78 & 2.74 \\
\hline
\end{tabular}




\begin{tabular}{lccccc}
\hline \multirow{2}{*}{ GARCH-N } & \multirow{2}{*}{ GARCH-T } & EGARCH- & EGARCH- & GARCH- & EGARCH \\
& & $N$ & $T$ & GPD & - GPD \\
\hline$U$ & & & & $1.62^{\prime}$ & $1.63^{\prime}$ \\
\hline$\xi$ & & & & $-0.0036^{\prime}$ & $0.064^{\prime}$ \\
\hline$\beta$ & & & $0.506^{\prime}$ & $0.4474^{\prime}$ \\
\hline
\end{tabular}

Source: Authors'calculation and deduction

“*”, "'” significance at $1 \%$, and $5 \%$ respectively

The stress-testing results are reported in TABLE 3 and plotted in the appendix for each market risk model (see FIGURES 1 - 6).

TABLE 3: Model stress testing results

\begin{tabular}{lcccc}
\hline $\begin{array}{c}\text { Market Risk } \\
\text { Model }\end{array}$ & $\begin{array}{c}\text { GARCH Volatility } \\
\text { Specification }\end{array}$ & Distribution Assumption & $\begin{array}{c}\text { Confi- } \\
\text { dence } \\
\text { level }\end{array}$ & $\begin{array}{c}\text { Number of } \\
\text { Violations }\end{array}$ \\
\hline GARCH - N & Symmetric & Normal distribution & $99 \%$ & 19 violations \\
\hline GARCH - T & Symmetric & Student's t distribution & $99 \%$ & 4 violations \\
\hline EGARCH - N & Asymmetric & Normal distribution & $99 \%$ & 17 violations \\
\hline EGARCH - T & Asymmetric & Student's t distribution & $99 \%$ & 3 violations \\
\hline GARCH - GPD & Symmetric & Generalized Pareto distribution & $99 \%$ & 6 violations \\
\hline EGARCH - GPD & Asymmetric & Generalized Pareto distribution & $99 \%$ & 4 violations \\
\hline
\end{tabular}

Source: Authors'calculation and deduction

From the results, one can conclude that the asymmetric GARCH specification (EGARCH) produces market risk models that are superior to symmetric GARCH models. This is clearly observed in TABLE 3: VaR models employing EGARCH volatility specifications have significantly fewer violations over the stress-testing period. GARCH specifications that fail to capture the leverage effect produce less accurate $\mathrm{VaR}$ measures. Clearly, distribution assumptions play an important role in VaR modelling. The risk models assuming normal distributions contain the highest number of violations of all estimated models. The normal distribution is clearly not applicable to daily JSE ALSI returns. The normal distribution fails to adequately capture information in the distribution tails, leading to inaccurate VaR measures. Risk models assuming a fat-tailed distribution, like the Student's t-test, perform strikingly better in estimating market risk. The same applies to the EVT models, their distribution tails being captured by the Generalized Pareto Distribution. Because of their adequate focus on the distribution tails, the risk models assuming Student's $t$ or Generalized Pareto distribution result in a significant reduction in the number of violations.

\section{CONCLUSION}

Recently, the Johannesburg Stock Exchange has become more volatile due to dynamic changes in market sentiment and investors' risk attitude. Since volatility is the main ingredient in value at 
risk estimation, the issue of how to forecast it accurately has received much attention. In this paper symmetric (GARCH) as well as asymmetric (EGARCH) volatility models were used under different distribution assumptions i.e. extreme value theory distributions, Generalized Error Distribution, Student's $t$, and normal distribution. The evidence shows that the distributional assumption plays an important role in forecasting volatility and predicting potential losses on the Johannesburg Stock Exchange. Using the JSE ALSI, the Student's t distribution has produced fewer violations in the out-sample period for both symmetric and asymmetric based market risk models. Therefore, the distribution assumption tends to play a more important role than the volatility specification in computing JSE ALSI value at risk estimates. These results suggest that JSE returns are more leptokurtic with few occurrences of extreme losses.

\section{LIST OF REFERENCES}

Bollerslev, T. (1986). Generalized Autoregressive Conditional Heteroskedasticity. Journa/ of Econometrics, 31 (3), pp. 307-327

Burns, P. (2002). The Quality of Value at Risk via Univariate GARCH. Working paper. [Online] Available: http://www.burns-stat.com. (Accessed 08/01/2011)

Fernandez, V. (2003). Extreme Value Theory and Value at Risk. Revista de Analisis Economico, 18(1), pp. 1-32.

Frey, R. and McNeil, A.J. (2000). Estimation of Tail-related Risk Measures for Heteroscedastic Financial Time Series: An Extreme Value Approach. Journal of Empirical Finance, 7, pp. 271-300.

Huang, C.T., Hung, J. \& Chang, C.M. (2010). The Roles of Distribution Assumption and Symmetric Specification of GARCH Model on VaR and Option Pricing. Journal of Money, Investment and Banking, 17 , pp. 37-46.

Kuester, K., Mittnik, S. and Paolella, M.S. (2006). Value-at-Risk Prediction: A Comparison of Alternative Strategies. Journal of Financial Econometrics, 4(1), pp. 53-89.

Muteba Mwamba, J. (2011). Predictability of Stock Price Behaviour in South Africa: A Non-parametric Approach. African Finance Journal, 13(1), pp.14-27.

Nelson, D.B. (1991). Conditional Heteroskedasticity in Asset Returns: A New Approach. Econometrica, 59 , pp. 347-370.

Nelson, D.B. (1992).: "Filtering and Forecasting with Misspecified ARCH Models I: Getting the Right Variance with the Wrong Model. Journal of Econometrics, 52, pp. 61-90.

Urbani, P. (2010): When Normality is not Enough. Working paper. [Online] Available:

http://www.edge-fund.com/UrbaniXX.pdf. (Accessed 09/01/2011). 


\section{APPENDIX}

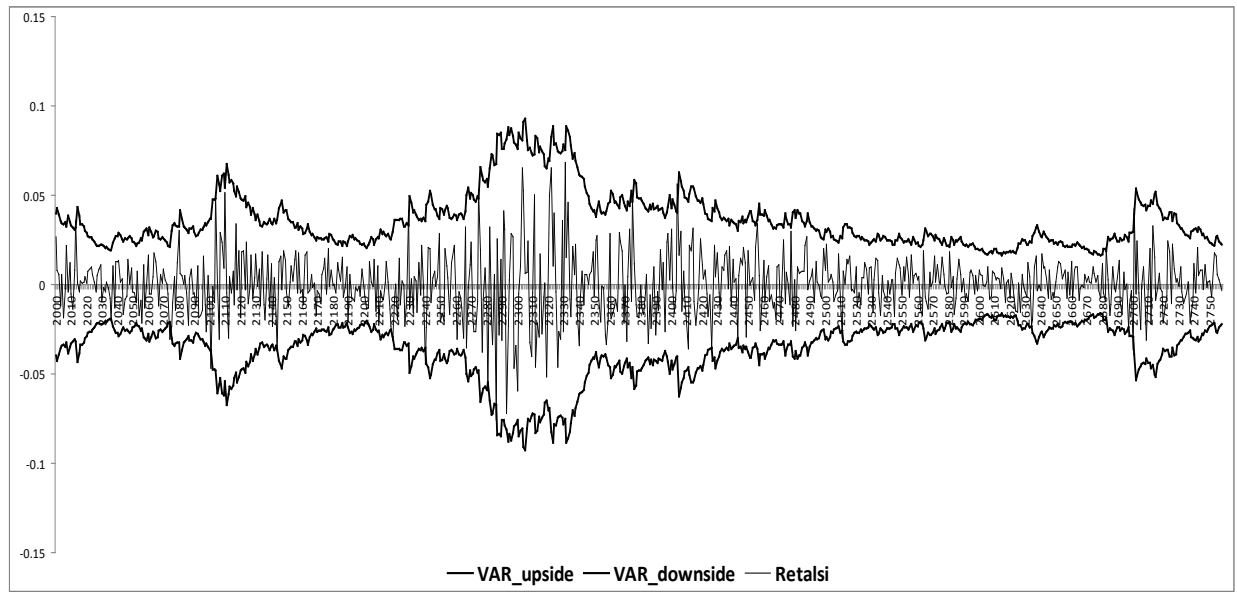

FIGURE 1: Garch-N

Source: Authors' calculations, based on JSE ALSI return series

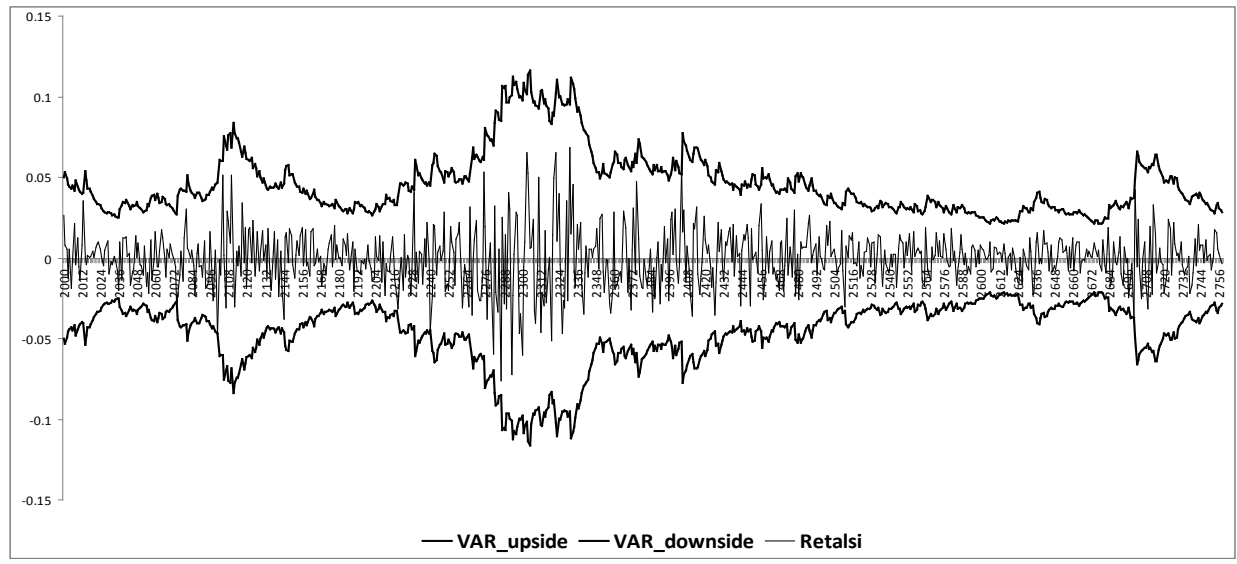

FIGURE 2: Garch-L

Source: Authors' calculations, based on JSE ALS/ return series 


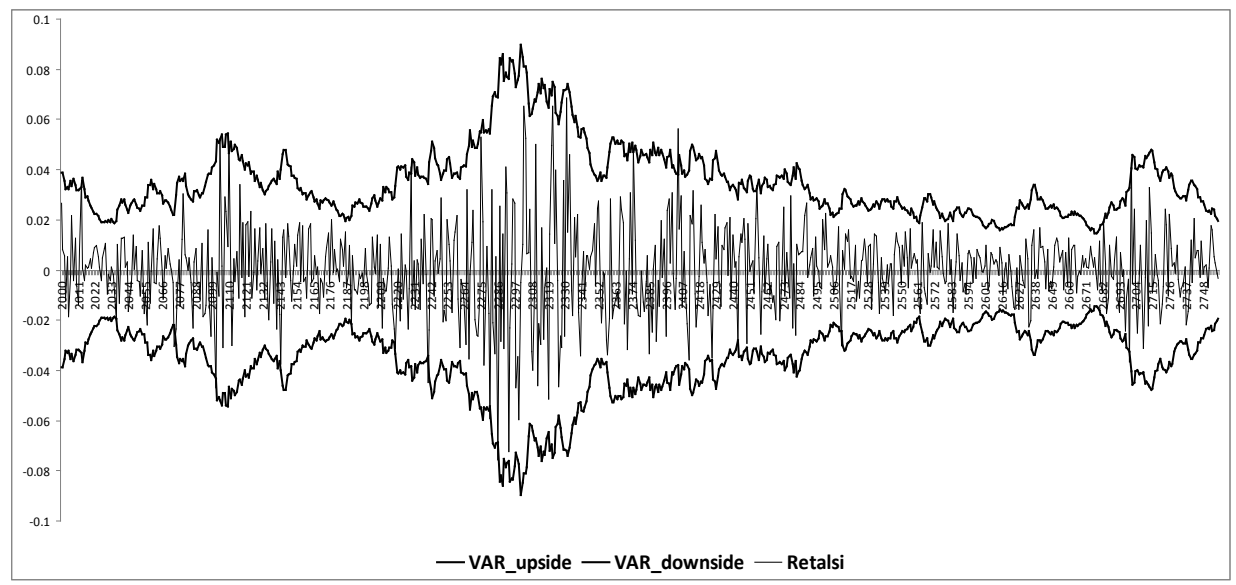

FIGURE 3: Garch-N

Source: $\quad$ Authors' calculations, based on JSE ALS/ return series

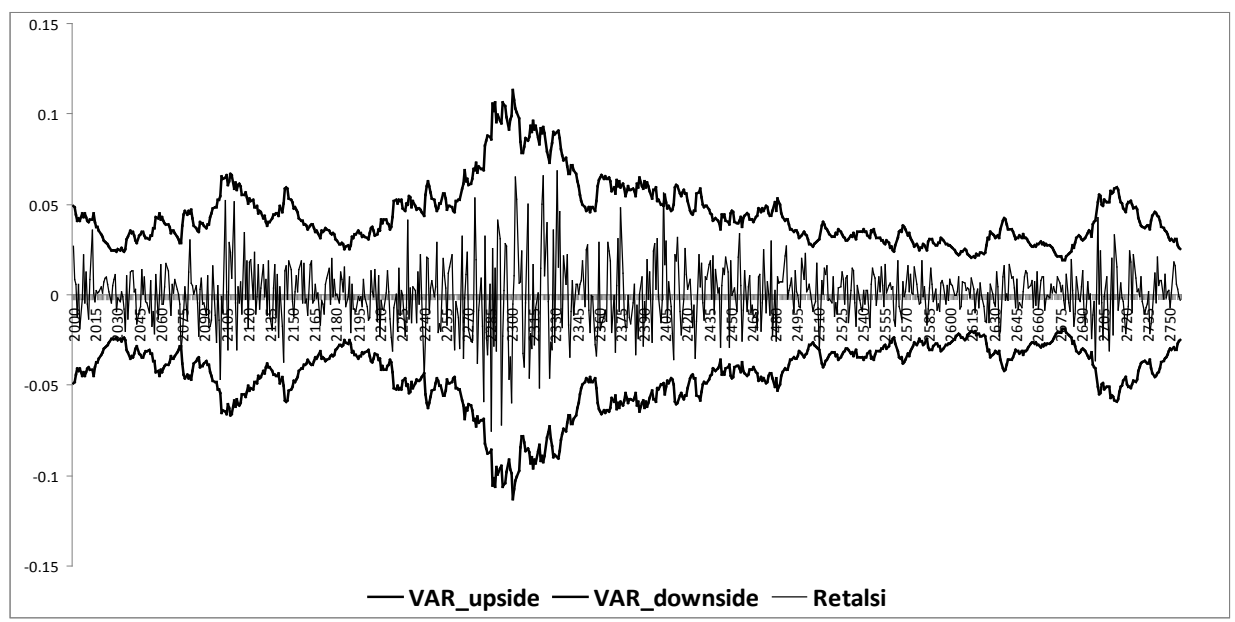

FIGURE 4: Egarch-T

Source: $\quad$ Authors' calculations, based on JSE ALS/ return series 


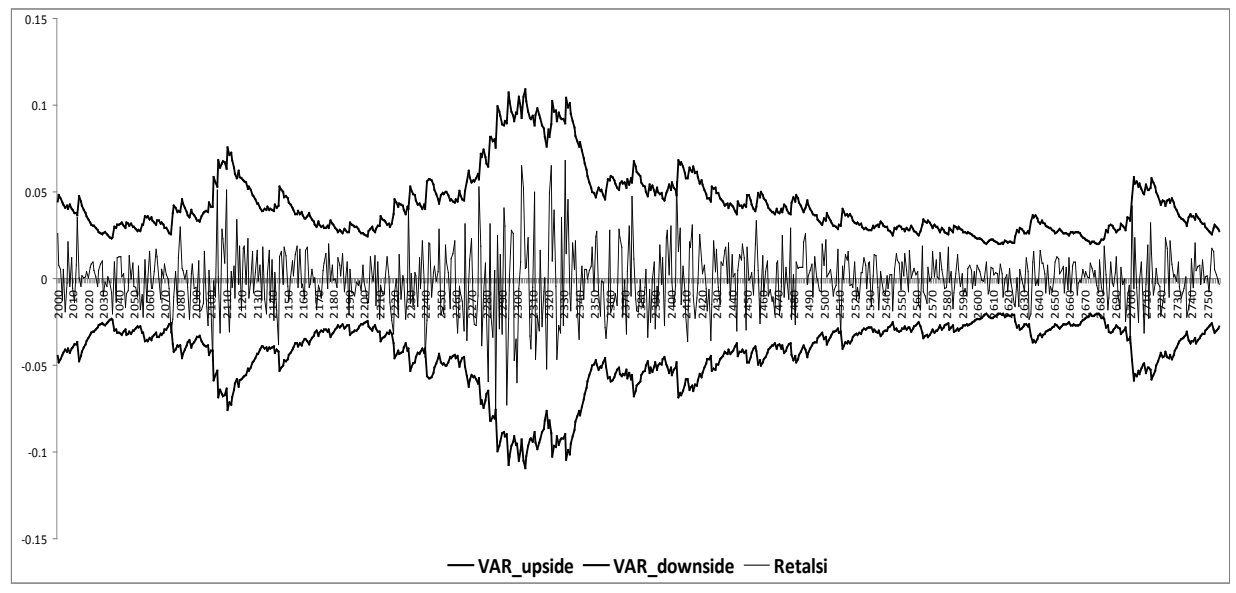

FIGURE 5: Garch-GPD

Source: $\quad$ Authors' calculations, based on JSE ALSI return series

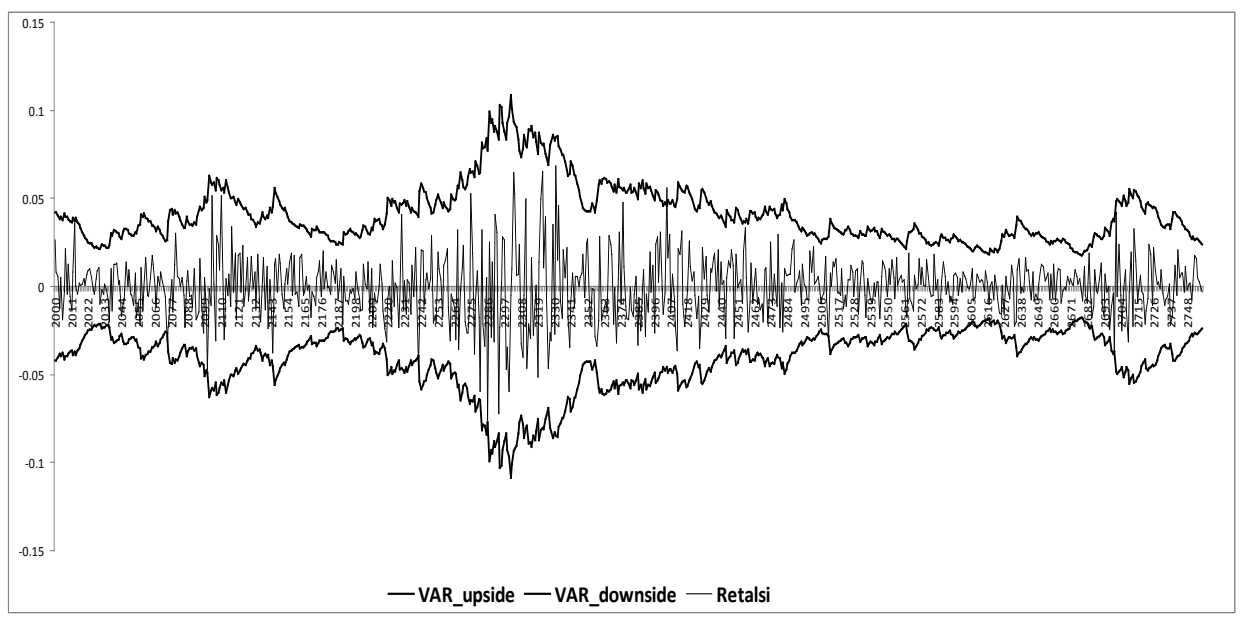

FIGURE 6: EGarch-GPD

Source: Authors' calculations, based on JSE ALS/ return series 


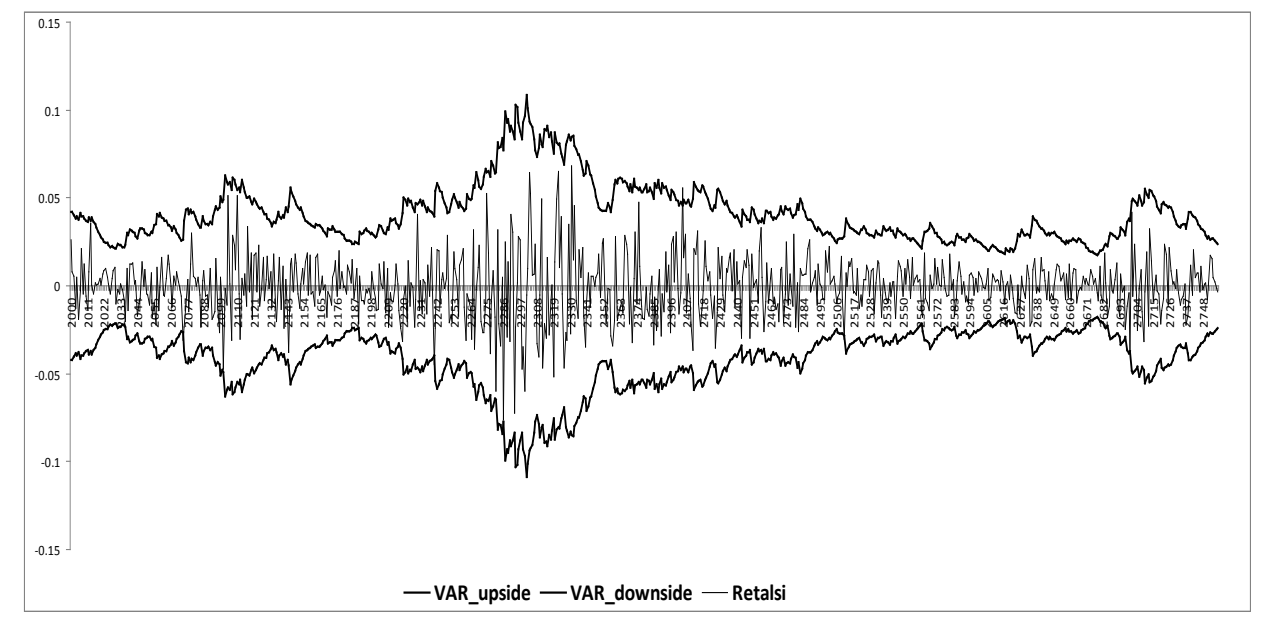

FIGURE 6: Egarch-GPD

Source: Authors' calculations, based on JSE ALSI return series 